\title{
Determining the Local Dark Matter Density
}

\author{
Hamish Silverwood* \\ GRAPPA, University of Amsterdam, Science Park 903, 1098 XH Amsterdam, The Netherlands \\ E-mail: h.g.m.silverwood@uva.nl
}

\section{Sofia Sivertsson}

GRAPPA, University of Amsterdam, Science Park 903, 1098 XH Amsterdam, The Netherlands, and The Oskar Klein Centre for Cosmoparticle Physics, Department of Physics, Stockholm University, AlbaNova, SE-106 91 Stockholm, Sweden

E-mail: sofiasiekth.se

\section{Pascal Steger}

Institute for Astronomy, Department of Physics, ETH Zürich, Wolfgang-Pauli-Strasse 27,

CH-8093 Zürich, Switzerland

E-mail: psteger@phys.ethz.ch

\section{Justin Read}

Department of Physics, University of Surrey, Guildford, GU2 7XH, Surrey, UK

E-mail: j.readesurrey.ac.uk

\section{Gianfranco Bertone}

GRAPPA, University of Amsterdam, Science Park 903, 1098 XH Amsterdam, The Netherlands

E-mail: gf.bertone@gmail.com

\begin{abstract}
Determination of the Dark Matter density at the solar position is critical to direct and indirect dark matter searches. Additionally, it is important to make this determination with as few assumptions as possible, as results from direct detection searches are used to explore a wide variety of theoretical models, and hidden astrophysical assumptions could bias theoretical searches. Here we present a Jeans analysis based method for the determination of the local dark matter density which allows us to limit the number of assumptions we need to make. We fit baryon and Dark Matter density models to tracer density and velocity dispersion data via integrated Jeans equations, and from these derive the local dark matter density. In this work we present the method and show tests on mock data.
\end{abstract}

The 34th International Cosmic Ray Conference,

30 July- 6 August, 2015

The Hague, The Netherlands

\footnotetext{
*Speaker.
} 


\section{Introduction}

The density of Dark Matter (DM) at the solar position is of great importance to a wide range of experiments. The local DM density, $\rho_{\mathrm{DM}}$, is an input to the calculation of event rates in direct DM detection experiments, and is degenerate with the DM-nucleon cross section [1]. Additionally the rate of neutrinos expected from DM annihilations in the Sun is also dependent on the local DM density $[?, 2,3,4,5]$. As these and other experiments are used to draw limits on DM phenomenology, the impact of incorrect or inaccurate local DM density measurements is felt beyond the realm of astrophysics.

The history of local DM measurements can be traced back to [6], and progress since then has been characterized not only by increasingly precise data, but also a reduction in systematics and modeling assumptions. As we move into the era of Gaia data starting in 2016, which promises high precision data on an unprecedented number of stars, the primary source of uncertainty on $\rho_{\mathrm{DM}}$ will be systematic. Thus a reduction in the strength and number of assumptions in our analysis techniques is crucial.

A well established method to determine $\rho_{\mathrm{DM}}$ is to measure the vertical ( $z$-direction) motions of tracer stars in the galactic disc plane $[7,8,9,10,11]$. This direction is particularly interesting as the baryonic contribution falls off rapidly, allowing one to probe the DM contribution which is approximately constant with height. In this proceedings we introduce a new method to determine the local DM density by fitting a mass model to tracer density and velocity dispersion of stars in the z-direction via the Jeans equations and present initial tests on mock data.

\section{Method}

The motions of a population of tracer stars can be modelled as a collisionless fluid, and thus described by the collisionless Boltzman equation. When integrated over velocity this yields a set of moment equations, known as the Jeans equation [12]. In axi-symmetric coordinates, and assuming dynamical equilibrium of the disc which allows us to eliminate any time derivatives, the $z$-direction Jeans equation becomes:

$$
\underbrace{\frac{1}{R v} \frac{\partial}{\partial R}\left(R v_{i} \sigma_{R z}\right)}_{\text {tilterm: } \mathscr{T}}+\frac{1}{v} \frac{d}{d z}\left(v \sigma_{z}^{2}\right)=\underbrace{-\frac{d \Phi}{d z}}_{K_{z}},
$$

where $v$ is the density of tracer stars in the vertical direction, $\sigma_{z}^{2}$ is the vertical velocity dispersion, and $\sigma_{R, z}^{2}$ is the cross term in the velocity dispersion tensor coupling vertical and radial motions. While Eq. 2.1 contains the necessary elements to fit a mass model to tracer density and velocity dispersion data, it also contains derivatives, which would amplify noise already present in the binned quantities $v, \sigma_{z}^{2}$, and $\sigma_{R, z}^{2}$. Thus we take Eq. 2.1 and integrate over $z$ to yield:

$$
\sigma_{z}^{2}(z)=\frac{1}{v(z)} \int_{z_{\min }}^{z} v\left(z^{\prime}\right)\left[K_{z}\left(z^{\prime}\right)-\mathscr{T}\left(z^{\prime}\right)\right] d z^{\prime}+\frac{C}{v(z)},
$$

where the normalisation parameter $C$ is fitted to data along with the other model parameters. As we have replaced numerical differentiation with integration this equation is more robust to noise in the data. 
The $K_{z}$ term can accomodate any theory of gravity, but for this work we use only the standard Newtonian weak field gravity, given by the Poisson equation

$$
\nabla^{2} \Phi=\frac{\partial^{2} \Phi}{\partial z^{2}}+\underbrace{\frac{1}{R} \frac{\partial V_{c}^{2}(R)}{\partial R}}_{\text {rotation curve term: } \mathscr{R}}=4 \pi G \rho
$$

where $V_{c}^{2}(R)$ is the circular speed curve at galactocentric radius $R$, and $\rho$ is the total (baryonic $+\mathrm{DM})$ matter density. For this study we assume a flat rotation curve, which means $\mathscr{R}$ goes to zero. Taking Eq. 2.3 and integrating yields

$$
\Sigma_{z}(z)=\frac{\left|K_{z}\right|}{2 \pi G}
$$

where $\Sigma_{z}(z)$ is the total surface mass density of the disc.

With these equations we can now describe how to derive the local DM density. We have (mock) data for the velocity dispersion $\sigma_{z}^{2}$ and the tracer density $v(z)$, derived from position and velocity measurements of tracer stars. This data is derived by binning, and the bin centres define a set of $z$ points. We then construct a model consisting of several elements, each described a number of parameters:

- Baryonic mass density is modelled as a simple exponential disk:

$$
\rho_{\text {baryon }}(z)=\frac{1}{4 \pi G}\left|\frac{K_{\mathrm{brn}} D_{\mathrm{brn}}^{2}}{\left(D_{\mathrm{brn}}^{2}+z^{2}\right)^{1.5}}\right|
$$

where $K_{\text {brn }}$ sets the mass of the disc, with dimensions of acceleration, and $D_{\text {brn }}$ sets the scale height of the disc, with dimensions of length. In future work a more realistic, data driven baryonic model will be implemented.

- Dark matter density is modelled using a combination of a term that is constant with height, and an additional 'dark disc' term, described with the same parameterisation as the baryonic disc, but with a much higher limit on the scale height parameter $D_{\mathrm{DD}}$ :

$$
\rho_{\mathrm{DM}}(z)=\rho_{\mathrm{DM}, \text { const. }}+\frac{1}{4 \pi G}\left|\frac{K_{\mathrm{DD}} D_{\mathrm{DD}}^{2}}{\left(D_{\mathrm{DD}}^{2}+z^{2}\right)^{1.5}}\right|
$$

The dark disc can be generated through several mechanisms, such as accretion of DM subhalos $[13,14,15]$, or the flattening of a spherical DM halo in the response to the formation of the baryon disc $[16,17,18]$. The dark disc element is turned on and off for various runs. More complex dark discs can be modelled as a sum of these dark disc terms.

- Tilt Term Deriving the tilt term would stricly require us to solve the full set of axisymmetric Jeans equations, but here we show that we are able to cope with this term even in the one dimensional approximation. Tracer density and velocity dispersion are assumed to follow an exponential in the $R$-direction:

$$
\begin{gathered}
v(R, z)=v(z) \exp \left(-R / R_{0}\right) \\
\sigma_{R z}(R, z)=\sigma_{R z}(z) \exp \left(-R / R_{0}\right),
\end{gathered}
$$


while $\sigma_{R z}$ is described by a power law, which gives a good fit to current data [?]:

$$
\sigma_{R z}(R, z)=\left.A z^{n}\right|_{R}
$$

This reduces the tilt term at the solar position to a three parameter model:

$$
\mathscr{T}\left(R_{\odot}, z\right)=\left.A z^{n}\right|_{R_{\odot}}\left[\frac{1}{R_{\odot}}-\frac{2}{R_{0}}\right]
$$

For some runs this term is set to zero, to assess the impact of ignoring the effects of tilt. Note that while here we describe the radial component of tracer density and velocity dispersion as an exponential, alternative descriptions would be possible if motivated by data.

- Tracer density is also modelled, even though we have access to this data. We do this so that the tracer density can vary within the uncertainty of the data if such a variation improves the overall fit of the model. To describe this element we go beyond the usual exponential disc model, and instead use a description of $v$ where the derivative of its natural log is allowed to vary from bin to bin. We take

$$
k_{z} \equiv-\frac{d \ln v}{d z}
$$

and at each bin centre a value of $k_{z}$ is selected, and then $v$ is derived via numerical integration. This description allows us to limit unphysical models and fitting to noise by selecting appropriate priors on $k_{z}$ values.

The sum of the parameters that go into the above model, plus the normalisation constant $C$ from Eq. 2.2, form an $N$-dimensional parameters space ${ }^{1}$. Specifying a point in this parameter space, i.e. giving numerical values to each of the parameters, gives us numerical values for each of the terms on the RHS of Eq. 2.2. Thus we can derive $\sigma_{R, z}^{2}$ for this parameter space point. The model values for $v(z)$ and $\sigma_{z}(z)$ are then compared to data using a $\chi^{2}$ test. We then use Multinest $[19,20,21]$ to explore the parameter space within appropriate priors and map the posterior distribution, allowing us to determine credible regions for each of the parameters.

\section{Mock Data}

To hone and verify the method we first test it on mock data. For this study the mock data is 'as good as it gets' [22], in the sense that we add no measurement error. Assuming an exponential tracer density, a baryonic mass profile as described by Eq. 2.5, and a dark matter mass profile as described by Eq. 2.6, we can derive a velocity dispersion profile $\sigma_{z}^{2}(z)$ using Eq. 2.2. We then draw a stellar position $z^{\prime}$ from the exponential tracer density, and then draw its velocity from a gaussian centered on $v_{z}=0$ and with variance of $\sigma_{z}^{2}\left(z^{\prime}\right)$. This process is repeated to produce data sets with various numbers of stars. Assuming different scale heights for the exponential tracer density allows us to generate different data sets representing sampling of thick and thin disc stars.

\footnotetext{
${ }^{1} N$ is the sum of the number of bins plus one (as we have $k_{z}$ values for nu at each bin centre plus a value at $z=0$ ), two baryon mass profile parameters, either one or three DM parameters depending on if the dark disc is used, another three parameters if a tilt term is used, plus one parameter for the constant $C$ from Eq. 2.2.
} 
To generate mock tilt data we take the same list of stellar positions and draw a value for $v_{z} v_{R}$ from a Gaussian centred on $v_{z} v_{R}=0$ with a variance of $\sigma_{R z}\left(R_{\odot}, z\right)$, as described by Eq. 2.9 with values for the $A, n$, and $R_{0}$ parameters motivated by results from [?].

\section{Results}

Figure 1 shows the reconstruction of a constant DM density with sampling sizes of $10^{3}, 10^{4}$, $10^{5}$ and $10^{6}$ stars. This is a basic case, with the mock data containing no tilt term and no dark disc. The light grey band indicates $95 \%$ credible region on the posterior likelihood, while the dark grey band indicates the $68 \%$ credible region, marginalizing over all other parameters. Naturally as the number of tracer stars is increased the credible regions shrink and closes in on the true value of $\rho_{D} M$, which is indicated by the green line.

Figure 2 (a) shows a mock data set with a tilt term reconstructed with the tilt term set to zero. The $K_{z}$ term of Eq. 2.2, a negative number, is forced to a more negative value to compensate for the tilt term being set to zero. This then necessitates a higher mass density, which leads to a higher reconstructed value of $\rho_{\mathrm{DM}}$.

Figure 2 (b) shows the ability of the method to find and fit to a dark disk. The mock data DM profile (green line) is contained within the $68 \%$ credible region.

\section{Conclusions}

Determining the local DM density with as few assumptions as possible is important not only to understand the structure of the galaxy but also to interpret and analyse the results of direct and indirect DM detection experiments. Here we have presented a method to do this, which has minimal assumptions about the global properties of the DM halo and the distribution of the tracer stars. We have also presented a number of preliminary tests of this method on mock data, showing that the determination of DM become more precise with increased sample size, the peril of ignoring the tilt term, and the ability of the method to reconstruct a dark disk. More extensive tests of the method are presented in a recent paper [23].

\section{References}

[1] L. Baudis, A review of direct WIMP search experiments, Nucl.Phys.Proc.Suppl. 235-236 (2013) 405-412.

[2] J. Silk, K. A. Olive, and M. Srednicki, The Photino, the Sun and High-Energy Neutrinos, Phys.Rev.Lett. 55 (1985) 257-259.

[3] L. M. Krauss, K. Freese, W. Press, and D. Spergel, Cold dark matter candidates and the solar neutrino problem, Astrophys.J. 299 (1985) 1001.

[4] T. Gaisser, G. Steigman, and S. Tilav, Limits on Cold Dark Matter Candidates from Deep Underground Detectors, Phys.Rev. D34 (1986) 2206.

[5] A. Gould, Direct and Indirect Capture of Wimps by the Earth, Astrophys.J. 328 (1988) 919-939.

[6] J. Kapteyn, First Attempt at a Theory of the Arrangement and Motion of the Sidereal System, Astrophys.J. 55 (1922) 302-328. 


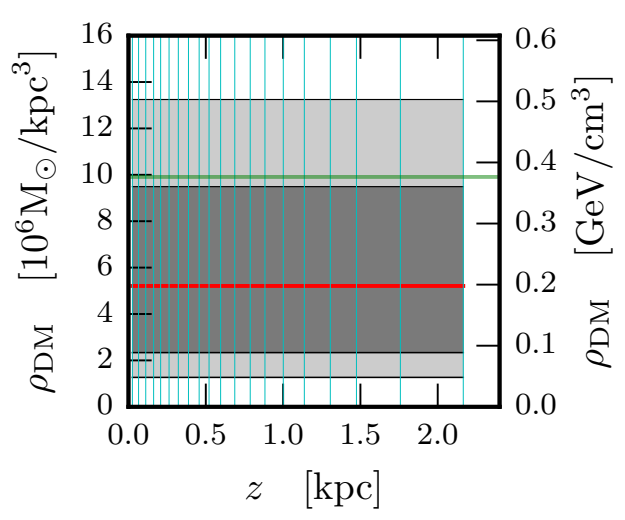

(a) $10^{3}$ tracer stars

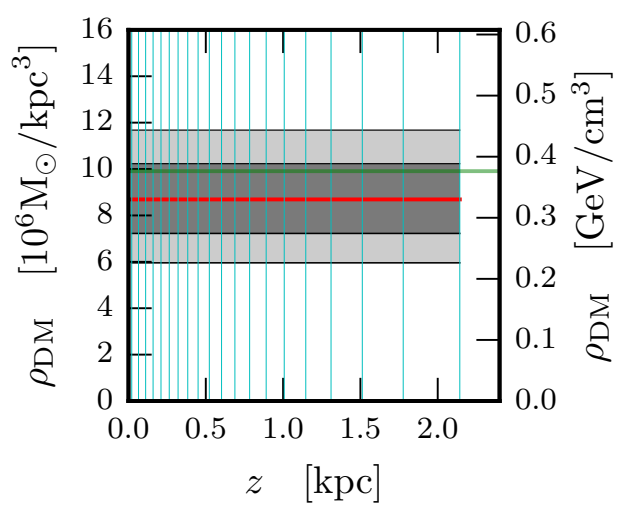

(c) $10^{5}$ tracer stars

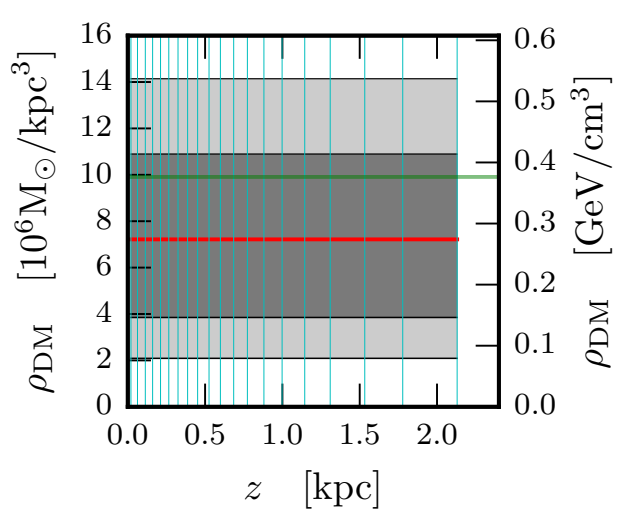

(b) $10^{4}$ tracer stars

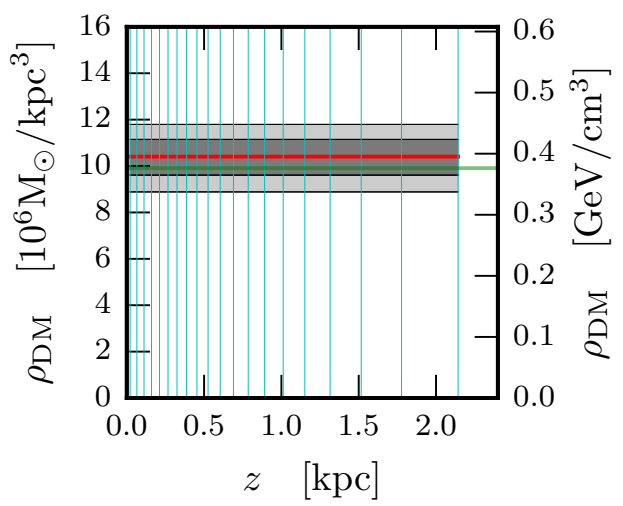

(d) $10^{6}$ tracer stars

Figure 1: Determination of DM density with various numbers of tracer stars. The value of $\rho_{\mathrm{DM}}$ used to generate the mock data is indicated by the green line. The light and dark grey bands indicate the $95 \%$ and $68 \%$ credible regions on the posterior likelihood, marginalizing over all other parameters. The red line shows the median value of the posterior.

[7] J. H. Oort, The force exerted by the stellar system in the direction perpendicular to the galactic plane and some related problems, Bulletin of the Astronomical Institutes of the Netherlands 6 (Aug., 1932) 249.

[8] K. Kuijken and G. Gilmore, The mass distribution in the galactic disc. I - A technique to determine the integral surface mass density of the disc near the sun., Mon.Not.Roy.Astron.Soc. 239 (Aug., 1989) 571-603.

[9] K. Kuijken and G. Gilmore, The Mass Distribution in the Galactic Disc - Part Two - Determination of the Surface Mass Density of the Galactic Disc Near the Sun, Mon.Not.Roy.Astron.Soc. 239 (1989) 605. 


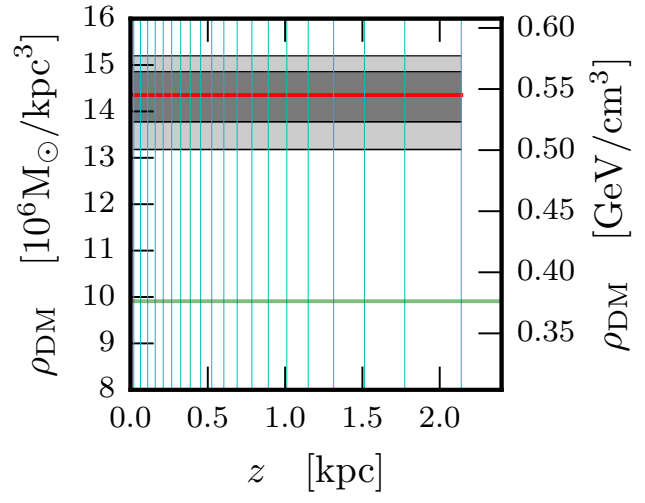

(a)

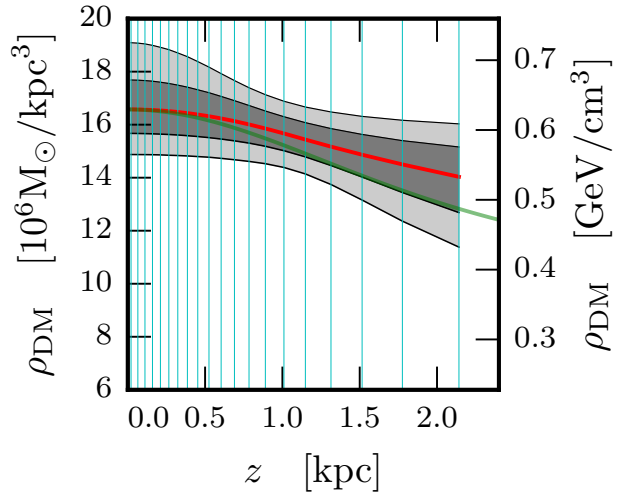

(b)

Figure 2: (a) Determination of $\rho_{\mathrm{DM}}$ using mock data with tilt but reconstructed using a model without a tilt term. This illustrates the danger of ignoring the tilt term. (b) Reconstruction a DM density profile incorporating a dark disc.

[10] J. N. Bahcall, Self-consistent determinations of the total amount of matter near the sun, "Astrophys.J." 276 (Jan., 1984) 169-181.

[11] J. N. Bahcall, $K$ giants and the total amount of matter near the sun, "Astrophys.J." 287 (Dec., 1984) 926-944.

[12] J. Binney and S. Tremaine, Galactic Dynamics. Princeton University Press, 2 ed., 2008.

[13] G. Lake, Must the disk and halo dark matter be different?, AJ 98 (Nov., 1989) 1554-1556.

[14] J. Read, G. Lake, O. Agertz, and V. P. Debattista, Thin, thick and dark discs in LCDM, Mon.Not.Roy.Astron.Soc. 389 (2008) 1041-1057, [arXiv: 0803.2714$].$

[15] J. Read, L. Mayer, A. Brooks, F. Governato, and G. Lake, A dark matter disc in three cosmological simulations of Milky Way mass galaxies, Mon.Not.Roy.Astron.Soc. 397 (2009) 44, [arXiv:0902.0009].

[16] N. Katz and J. E. Gunn, Dissipational galaxy formation I: Effects of gas dynamics, Astrophys. J. (1991).

[17] J. Dubinski, The Effect of dissipation on the shapes of dark halos, Astrophys.J. 431 (1994) 617-624, [astro-ph/9309001].

[18] V. P. Debattista, B. Moore, T. R. Quinn, S. Kazantzidis, R. Maas, et al., The Causes of Halo Shape Changes Induced by Cooling Baryons: Disks Versus Substructures, Astrophys.J. 681 (2008) 1076, [arXiv:0707.0737].

[19] F. Feroz and M. Hobson, Multimodal nested sampling: an efficient and robust alternative to MCMC methods for astronomical data analysis, Mon.Not.Roy.Astron.Soc. 384 (2008) 449, [arXiv:0704.3704].

[20] F. Feroz, M. Hobson, and M. Bridges, MultiNest: an efficient and robust Bayesian inference tool for cosmology and particle physics, Mon.Not.Roy.Astron.Soc. 398 (2009) 1601-1614, [arXiv:0809.3437]. 
[21] F. Feroz, M. Hobson, E. Cameron, and A. Pettitt, Importance Nested Sampling and the MultiNest Algorithm, arXiv:1306.2144.

[22] J. Read, The Local Dark Matter Density, J.Phys. G41 (2014) 063101, [arXiv: 1404.1938 ].

[23] H. Silverwood, S. Sivertsson, P. Steger, J. I. Read, and G. Bertone, A non-parametric method for measuring the local dark matter density, ArXiv e-prints (July, 2015) [arXiv: 1507.08581]. 